\title{
REDES COOPERATIVAS DE PESQUISA TECNOLÓGICA NA INDÚSTRIA DO PETRÓLEO OFFSHORE E O CASO DO BOMBEIO SUBMARINO: UMA ANÁLISE BIBLIOMÉTRICA
}

\author{
MATHEUS GONÇALVES DA SILVA PERETA \\ Graduando de Ciências Sociais na Universidade Estadual de Campinas, email: \\ matheusgoncalvessp@gmail.com
}

ANDRÉ TOSI FURTADO

Professor Titular do Departamento de Política Científica e Tecnológica na Universidade Estadual de Campinas, email: furtado@ige.unicamp.br

\begin{abstract}
ÁREA ABEIN: 06 - Redes de Inovação - Alianças de P\&D, Interações Universidade-Empresa, Cadeias. JEL - CODE: 030, 031, 032.
\end{abstract}

\section{RESUMO}

Este artigo explora as redes de cooperação de pesquisas tecnológicas para o desenvolvimento de novos conhecimentos sobre tecnologias emergentes no setor de petróleo e gás natural offshore. O principal desafio de nossa investigação é identificar as organizações que lideram a produção de novos conhecimentos sobre bombeio submarino, em sua fase pré-competitiva. Para superá-lo, identificamos artigos e trabalhos em anais de congresso que tratavam do bombeio submarino aplicado à situação específica da indústria do petróleo offshore numa base de dados e analisamos as informações através de uma perspectiva bibliométrica. Desse modo, foi possível construir indicadores que desenham a evolução tecnológica e o padrão de cooperação das organizações associadas ao bombeio submarino. Apoiamo-nos na literatura que trata de redes de inovação e da análise quantitativa em ciência e tecnologia para elaborar as análises bibliométricas realizadas. Por sua vez, a literatura técnica dos conceitos tecnológicos de bombas submarinas é consultada para fins de comparação técnico-econômica. Por tratar-se de pesquisa ainda em curso, o texto apresenta carácter mais indicativo do que conclusivo.

Palavras-chave: Inovação tecnológica. Indústria do petróleo. Redes de Inovação. Alianças de P\&D. Bibliometria.

\begin{abstract}
This paper explores the technological research cooperation networks for the development of new knowledges in emerging technologies in the offshore oil and gas industry. The main challenge of our research is to identify the organizations spearheading the production of new knowledges on subsea pumping in its pre-competitive stage. To overcome it, we identify articles and papers in conferece proceedings dealing subsea pumping applied to the specific situation of the offshore oil and gas industry in a database. Then, we analyze the data through a bibliometric approach. In this way it was possible to build indicators that draw the evolution of this technologies and the pattern of cooperation among organizations associated to subsea pumping. We use the literature on networks of innovation and quantitative analysis in science and technology to develop the bibliometric analyzes. Furthermore, the technical literature on performance of technological concepts of subsea pumps is consulted for the purpose of technic-economic evaluation. This paper deals an ongoing research, so the final remarks are more indicative than conclusive.
\end{abstract}

Keywords: Technicological innovation. Oil and gas industry. Networks of innovation. R\&D Alliances. Bibliometrics. 


\section{INTRODUÇÃO}

A participação dos hidrocarbonetos na matriz energética mundial se manterá a uma proporção de 30\% até meados de 2030 (BP, 2015), implicando na necessidade da indústria do petróleo explorar regiões geológicas cada vez mais complexas. Por se tratar de um recurso natural escasso, as empresas do setor devem desenvolver métodos de extração e recuperação de petróleos economicamente viáveis que maximizem a rentabilidade da produção desse energético em um ambiente internacional caracterizado pela acentuada oscilação dos preços dessa commodity. No caso específico do segmento offshore, as companhias de petróleo investem em tecnologias emergentes cooperando com empresas fornecedoras de equipamentos e serviços e universidades com o objetivo de esticar suas capacidades operacionais em lâminas d'água cada vez maiores.

A complexidade envolvida nas atividades dessa indústria demanda um nível de capacitação tecnológica das organizações para manter o ritmo de produção de conhecimentos e a difusão de inovações mais performantes; assim sendo, poucas empresas, majoritariamente de países desenvolvidos, figuram como importantes players na fronteira tecnológica do segmento. Apesar disso, o aumento dos custos das atividades de pesquisa e inovação em consonância aos riscos e incertezas econômicas que envolvem a difusão de novas tecnologias vem reorganizando o padrão de relacionamento das organizações desde seu estágio pré-competitivo. O estabelecimento de alianças estratégicas entre diferentes organizações, especialmente, companhias de petróleo, empresas fornecedoras de bens e serviços, instituições de pesquisa e universidades acabou por caracterizar relações do tipo usuário-fornecedor que se expandem globalmente por toda a cadeia produtiva relacionada a essa indústria. Desse modo, novas empresas de países em desenvolvimento com uma base tecnológica consolidada (FURTADO; FREITAS, 2000; 2001) começaram a absorver conhecimentos tecnológicos que as tornaram habilitadas a participar de maneira ativa no processo de inovação.

O caso do bombeio submarino é ilustrativo nesse sentido, pois essas tecnologias e métodos de recuperação e desenvolvimento da produção de hidrocarbonetos são necessários para permitir a exploração de óleo com menor saturação de resíduos, maximizando o tempo de vida útil dos campos petrolíferos e aumentando a rentabilidade das atividades dessa indústria em alturas de lâminas d'água cada vez maiores. Por se tratarem de tecnologias emergentes ainda em estágio pré-competitivo, os riscos de custos e de fabilidade desses sistemas tecnológicos ainda são altos. Dessa maneira, as organizações envolvidas com o desenvolvimento do bombeio submarino vêm cooperando a fim de produzir novos conhecimentos que possam provocar descontinuidades no atual paradigma tecnológico do setor de exploração e produção de petróleo e gás natural offshore.

O trabalho está organizado em três seções, além desta introdução. A primeira seção diz respeito à metodologia aplicada para coletar e depurar os dados em um banco de dados, assim como uma comparação técnica e do design dos conceitos tecnológicos de bombeamento submarino trabalhados neste artigo. Na segunda seção são caracterizadas as informações contidas nos artigos e trabalhos em anais de congressos a respeito das tecnologias de bombeio submarino, enfatizando suas origens geográficas e institucionais. Na terceira seção se discute as publicações em cooperação afiliadas a mais de uma organização, com o objetivo de identificar quais empresas cooperam para produzir mais conhecimentos a respeito do bombeio submarino e os nós dessa rede de cooperação. Algumas considerações finais encerram o debate promovido por este trabalho.

\section{METODOLOGIA}

Este trabalho é derivado dos resultados parciais de uma pesquisa de iniciação científica ainda em andamento. Seis passos metodológicos foram percorridos para encontrar os resultados que satisfazem os interesses de nossa investigação, a saber, i) levantamento de projetos de bombeio submarino em periódicos especializados a fim de identificar quais desses conceitos tecnológicos são mais utilizados na indústria do petróleo offshore; ii) sistematização de uma comparação técnico-econômica das tecnologias levantadas no passo anterior, através de revisão da literatura técnica; iii) elaboração de queries relacionadas às tecnologias de bombeio submarino para levantar dados de publicações científicas em um banco de dados; iv) coleta de dados na plataforma Scopus; v) depuração dos dados com a finalidade de se 
chegar a uma amostra condizente com os objetivos da pesquisa e, finalmente, vi) análise bibliométrica dos dados de publicações científicas sobre tecnologias de bombeio submarino, enfatizando as informações a respeito de cooperações entre organizações que publicam em co-autoria.

A metodologia de pesquisa empregada em nossa investigação visou, em um primeiro momento, identificar quais tecnologias de bombeio submarino vêm sendo desenvolvidas e aplicadas na indústria do petróleo offshore. Tendo como aporte o survey de equipamentos subsea realizado pelo periódico Offshore Magazine e disponibilizado em 2015, foi possível elencar 4 sistemas tecnológicos (Tecnologias Submarinas de Compressão de Gases; Tecnologias Submarinas de Levantamento Artificial; Tecnologias Submarinas de Injeção de Água e, Tecnologias Submarinas de Separação) e 5 tipos de bombas submarinas (Bombas Multifásicas; Bombas de Duplo-Parafuso; Bombas Helico-Axiais; Bombas Centrífugas e, finalmente, Bombas Elétricas Submersíveis). A fim de compreender as diferenças técnicas entre esses conceitos tecnológicos, realizamos uma analise comparativa dos mesmos, nos apoiando em estudos técnico-econômicos e trabalhos acadêmicos.

\subsection{UMA BREVE COMPARAÇÃO ENTRE OS PRINCIPAIS CONCEITOS TECNOLÓGICOS ASSOCIADOS AO BOMBEIO SUBMARINO APLICADO À INDÚSTRIA DO PETRÓLEO OFFSHORE}

As metodologias de bombeio submarino associadas ao aumento da produtividade dos poços produtores se segmentam em duas vertentes, a saber, i) técnicas de aumento do fator de recuperação de petróleo e, ii) levantamento artificial de hidrocarbonetos. O primeiro grupo diz respeito à injeção de fluídos em um poço injetor a fim de modificar as condições físico-químicas do poço produtor, isto é, aumentando os níveis de pressão, diminuindo a tensão interfacial ou a viscosidade do petróleo. Por sua vez, o segundo grupo trata de métodos de mudança das condições dos fluídos, permitindo que atinjam a superfície.

O critério de seleção das tecnologias de bombeamento para aplicação subsea no segmento offshore da indústria do petróleo permanece, de certo modo, ainda relativo, pois depende dos dados de performance dos conceitos oferecidos pelas empresas fornecedoras, que podem ser manipulados para dar preferência a um conceito tecnológico em detrimento a outro (ROMERO; HUPP, 2013). O uso dos métodos de bombeio submarino aplicados no offshore tem se tornado uma opção mais comum na produção de petróleo de baixo $\mathrm{API}^{1}$, pois apresentam melhor eficiência operacional e maior taxa de produtividade nas mesmas condições de aplicação em comparação a outros métodos de levantamento artificial.

O bombeamento submarino em fase-única já não mais atende às necessidades econômicas e operacionais da recuperação de petróleo, devido aos seguintes fatores, i) um volume significativo de petróleo em bacias maduras é difícil de ser acessado, sobretudo, quando encalhados em locais não tradicionais à exploração pela indústria de petróleo offshore; ii) no desenvolvimento de grandes campos petrolíferos, o processo de "desgasificação" envolve o dispêndio de muitos recursos e um grande esforço operacional; iii) o aumento das distâncias entre o poço produtor e o continente, assim como da altura da lâmina d'água exige um aumento da resistência das tecnologias que realizam o escoamento da produção, sobretudo em ambientes hostis. O bombeamento multifásico, por sua vez, emerge como importante conceito tecnológico. As principais vantagens da produção multifásica em relação à monofásica são i) a aceleração da produção devido à diminuição do repuxo de pressão; ii) início e estabilização do fluxo de hidrocarbonetos que não podem ser escoados naturalmente até as unidades de produção; iii) aumento da distância operacional (lâmina d’água, distância entre o poço produtor e as unidades de produção); iv) redução dos custos operacionais e de intervenção nos poços; v) redução dos custos de desenvolvimento da produção subsea; vi) environmentally friendly - O processo de bombeamento submarino multifásico utiliza técnicas de captura de gases poluidores reinjetados aos poços para promover o aumento de pressão e consequente aumento do fator de recuperação de óleo e, finalmente, vii) Facilita a atividade de

\footnotetext{
${ }^{1}$ American Petroleum Institute Gravity ou API Gravity mensura quanto mais leve, ou pesada uma amostra de petróleo é em relação à água. Isto é, trata-se de uma medida inversamente proporcional à densidade, pois quanto maior o valor de API, menor a densidade do óleo.
} 
exploração e produção offshore em ambientes geológicos complexos (HUA et al., 2012; FALCONE et al., 2002).

O conceito de boosting está diretamente associado ao aumento do fator de recuperação de petróleo, através da instalação de bombas submersas, em poços falsos ou na cabeça dos poços, implicando no significativo aumento da pressão dos fluídos escoados até a superfície. As bombas relacionadas à separação de fluídos objetivam desagregar resíduos arenosos, parafinas, bem como água, gases e demais elementos contaminantes misturados ao petróleo através do uso de separadores. O bombeio centrifugo é semelhante ao conceito de bombeamento multifásico e de separação diferindo desses por instalar as bombas no interior dos poços produtores de petróleo (FURTADO; FREITAS, 2001).

Os dois conceitos de bombas submarinas mais utilizadas para operações subsea são do tipo twin-screw pump (TSP) e helico-axial pump (HAP). As TSPs são ideais para tratar de fluídos de alta viscosidade, todavia não são muito adequadas para lidar com resíduos sólidos, pois necessitam ser desmontadas em caso de sand trap. Além disso, seu design é específico para operar a condições pré-determinadas; isto quer dizer que com o passar do tempo, a diminuição do volume de petróleo e a alteração das condições físico-químicas no interior do poço limita a performance dessa tecnologia. As HAPs tem um princípio operacional similar às demais bombas centrífugas, isto é, aumentar a energia cinética acumulada em seus impulsores rotativos, convertendo-a em energia potencial e transferindo-a aos fluídos através de difusores estáticos (HUA et al., 2012).

Para aplicações de bombeio submarino na boca dos poços, o conceito subsea electrical submersible pump (ESP) é uma das opções tecnológicas mais empregadas na indústria do petróleo. As ESPs são utilizadas para complementar a energia necessária para elevar o fluxo de fluídos desde o poço produtor às instalações de produção. Seu princípio de operação se baseia na transmissão de energia elétrica ao ponto em que a ESP é instalada através de um cabo de alimentação. Nesse ponto, a energia elétrica é convertida em energia mecânica pelo motor conectado diretamente ao eixo da bomba. Embora anos de investimentos e melhorias de tecnológicas tenha sido aplicada no desenvolvimento das ESPs, essa tecnologia ainda apresenta taxas relativamente altas de falibilidade, devido, principalmente, a limitação de operacionalidade dos componentes elétricos empregados em ambientes hostis, sobretudo, no offshore profundo (ROMERO; HUPP, 2013).

\subsection{COLETA E DEPURAÇÃO DOS DADOS}

As informações sobre publicações associadas ao bombeio submarino aplicado na indústria do petróleo offshore foram coletadas na base de dados Scopus. Esta, por sua vez, possui o maior banco de dados de resumos e citações da literatura especializada, isto é, periódicos científicos, livros e anais de congressos nos campos da ciência, tecnologia, medicina, ciências sociais, artes e ciências humanas. Além disso, possui um conjunto de ferramentas próprias desenvolvidas para identificar, analisar e apresentar resultados de pesquisa de maneira sofisticada.

Em números, o acervo da base de dados Scopus conta com mais de 60 milhões de registros, 21.500 periódicos especializados; 360 publicações de mercado; mais de 530 séries de livros e aproximadamente 7,2 milhões de anais de congressos de cerca de 80.000 eventos diferentes pelo mundo. Também estão disponíveis na base, mais de 27 milhões de patentes de cinco escritórios diferentes (ELSEVIER, 2016). O tamanho expressivo do acervo da base Scopus foi um fator fundamental para a escolha de levantar dados que satisfizessem os interesses de nossa investigação, sobretudo por nosso objeto de estudo ser multidisciplinar, exigindo a coleta de informações de fontes diversificadas e em diferentes campos do conhecimento.

O acervo de informações sobre publicações disponível na base Scopus atende a diferentes perfis de pesquisadores e interesses institucionais. Ao considerar as instituições acadêmicas (I); governos e agências de fomento à pesquisa (II); instituições de P\&D (III) e, finalmente, organizações de 
ranqueamento $^{2}$ (IV) como os principais conjuntos de instituições que realizam consultas a essa base de dados, temos os seguintes perfis de pesquisadores e as principais contribuições à pesquisa oferecida pelas ferramentas da base Scopus.

\section{Tabela 1 - Perfis de pesquisadores da base de dados Scopus}

\begin{tabular}{|c|c|c|c|c|c|}
\hline & \multirow[b]{2}{*}{ Vantagens da Base Scopus ao Pesquisador } & \multicolumn{4}{|c|}{ Grupos Institucionais } \\
\hline $\begin{array}{c}\text { Perfil do } \\
\text { Pesquisador }\end{array}$ & & $\mathbf{I}$ & II & III & IV \\
\hline $\begin{array}{c}\text { Pesquisadores e } \\
\text { Autores }\end{array}$ & $\begin{array}{l}\text { Pesquisar tópicos/artigos na literatura especializada; Explorar a rede de } \\
\text { citações de publicações a fim de analizá-las; Identificar métricas de } \\
\text { publicações de um campo de pesquisa. }\end{array}$ & $\mathrm{X}$ & $\mathrm{X}$ & $\mathrm{X}$ & \\
\hline $\begin{array}{c}\text { Editores e } \\
\text { Revisionistas }\end{array}$ & $\begin{array}{l}\text { Monitorar tendências de pesquisas sobre um determinado tema na } \\
\text { literatura; Avaliar trabalhos anteriores de um determinado autor; } \\
\text { Encontrar e avaliar referências bibliográficas para elaborar um parecer } \\
\text { sobre trabalhos submetidos a periódicos. }\end{array}$ & $\mathrm{X}$ & $\mathrm{X}$ & & \\
\hline $\begin{array}{l}\text { Educadores e } \\
\text { Estudantes }\end{array}$ & $\begin{array}{l}\text { Encontrar informações complementares ao material didático; Pesquisar } \\
\text { literatura especializada que auxilie no processo de desenvolvimento de } \\
\text { trabalhos científicos e teses. }\end{array}$ & $\mathrm{X}$ & & & \\
\hline Bibliotecários & $\begin{array}{l}\text { Prover os usuários das bibliotecas com um banco de dados de grande } \\
\text { acervo de informações sobre resumos e citações de publicações nas mais } \\
\text { variadas áreas; Analisar as publicações mais citadas, influenciando, dessa } \\
\text { maneira, na tomada de decisões acerca das coleções das bibliotecas. }\end{array}$ & $\mathrm{X}$ & $\mathrm{X}$ & $\mathrm{X}$ & \\
\hline Administradores & $\begin{array}{l}\text { Acessar a produtividade acadêmica de um pesquisador ou de uma } \\
\text { instituição acadêmica; Tomar decisões de alocação de recursos baseados } \\
\text { na produtividade e impacto das pesquisas de um indivíduo ou instituição. }\end{array}$ & $\mathrm{X}$ & $\mathrm{X}$ & & \\
\hline $\begin{array}{c}\text { Gerentes de } \\
\text { Pesquisa }\end{array}$ & $\begin{array}{l}\text { Acelerar o processo de avaliação dos resultados da P\&D, aumentando a } \\
\text { produtividade e a efetividade da circulação dos conhecimentos } \\
\text { divulgados; Facilitar o processo de tomada de decisões baseadas em } \\
\text { informações científicas.; Avaliar as virtudes e fraquezas das experiências } \\
\text { dos competidores divulgadas nas publicações. }\end{array}$ & & & $\mathrm{X}$ & \\
\hline Rankings & Fornecer indicadores bibliométricos. & $\mathrm{X}$ & & & $\mathrm{X}$ \\
\hline
\end{tabular}

Fonte: Elsevier, 2016.

O método de consulta da Scopus se baseia em operadores booleanos ${ }^{3}$; assim sendo, os resultados de pesquisa são responsivos aos interesses do pesquisador ao retornar informações de publicações específicas às buscas realizadas. Essa característica justifica o interesse de nossa investigação em utilizar essa base de dados, pois, no processo de elaboração das queries, limitamos os resultados da pesquisa às palavras-chave a fim de identificar o conjunto de publicações que tratavam da aplicação de conceitos tecnológicos de bombeio submarino à situação específica da indústria do petróleo offshore.

A identificação dos conceitos tecnológicos, com base no survey de 2015 elaborado pela Offshore Megazine, orientou a formulação das queries utilizadas na base de dados com a finalidade de coletar publicações em periódicos e anais de eventos associados ao bombeio submarino. Assim sendo, foram elaboradas as seguintes queries: Subsea Gas Compression Technology; Subsea Boosting Technology; Subsea Water Injection Technology; Subsea Separation Technology; Subsea Multiphase Pump; Subsea Twin-Screw Pump; Subsea Helico-Axial Pump; Subsea Centrifugal Pump e, finalmente, Electrical Submersible Pump. A primeira depuração dos dados indicou a necessidade em diferenciá-los, conceitualmente, em dois grupos, a saber, i) sistemas submarinos; ii) bombas submarinas. O primeiro

\footnotetext{
${ }^{2}$ Organizações de raqueamento são empresas especializadas na sistematização, avaliação e venda de indicadores quantitativos e qualitativos baseados no ranqueamento de suas amostras.

${ }^{3}$ Operadores booleanos são instrumentos de pesquisas que permitem a palavras, ou grupos de palavras serem combinadas de maneiras diferentes de modo a influenciar os resultados de uma pesquisa numa base de dados.
} 
grupo diz respeito às publicações que tratam dos equipamentos e sistemas de produção complementares ao bombeio submarino, mas que não se limitam à performance, ou especificações técnicas das bombas. Por sua vez, o segundo grupo de publicações trata exclusivamente dos conceitos tecnológicos de bombas submarinas.

O próximo passo foi coletar dados de publicação a partir das queries na base de dados Scopus. Inicialmente, foram encontrados 390 resultados para as consultas realizadas; todavia, os mecanismos de busca de informações nessa base de dados permitem limitar os resultados a fim de filtrá-los de acordo com a finalidade da pesquisa. Desse modo, limitamos, em um primeiro momento, os resultados aqueles que incluíam as seguintes palavras-chave - subsea technology; subsea production system; ou, subsea pumping. A escolha dessas palavras-chave se justifica por selecionarem as publicações que tratam centralmente as tecnologias de bombeio submarino.

Dando prosseguimento à depuração dos dados, limitamos os resultados a apenas artigos, ou anais apresentados em conferências, pois dizem respeito a publicações com característica de apresentar conhecimentos e experiências inéditos às partes interessadas nos projetos tecnológicos, tais quais, empresas, instituições acadêmicas e agências governamentais. A opção metodológica por considerar apenas artigos e anais de congressos foi feita tomando nota que é comum à área das engenharias e tecnológicas apresentar resultados de performances em eventos, pois facilita o acesso das empresas aos conhecimentos e tecnologias existentes em um nível pré-competitivo. O próximo recorte foi direcionado à exclusão dos resultados repetidos no interior de cada query.

Todavia, foram identificadas publicações repetidas entre as queries. Considerando, dessa maneira, o conjunto de publicações inéditas associadas ao bombeio submarino aplicado ao segmento offshore da indústria do petróleo encontrado foi de 111 artigos ou trabalhos em anais de congressos entre 1988 e 2015.

Tabela 2 - Número de publicações sobre bombeio submarino por etapas de depuração dos dados na base Scopus

\begin{tabular}{|c|c|c|c|c|c|}
\cline { 2 - 5 } \multicolumn{1}{c|}{ Queries } & \multicolumn{4}{c|}{ Número de Documentos } \\
\cline { 2 - 6 } & $\begin{array}{c}\text { Resultados } \\
\text { Iniciais }\end{array}$ & $\begin{array}{c}\text { Limitados às } \\
\text { Palavras-Chave }\end{array}$ & $\begin{array}{c}\text { Limitados a } \\
\text { Artigos e } \\
\text { Trabalhos em } \\
\text { Anais }\end{array}$ & $\begin{array}{c}\text { Não Repetidos } \\
\text { no Interior } \\
\text { das Queries }\end{array}$ \\
\hline \multirow{3}{*}{$\begin{array}{c}\text { Sistemas } \\
\text { Tecnológicos }\end{array}$} & $\begin{array}{c}\text { Subsea Boosting Technology } \\
\text { Technology }\end{array}$ & 32 & 31 & 19 & 11 \\
\cline { 2 - 6 } & Subsea Water Injection Technology & 74 & 44 & 22 & 20 \\
\hline
\end{tabular}




\begin{tabular}{|c|c|c|c|c|c|}
\hline & Subsea Separation Technology & 79 & 64 & 53 & 31 \\
\hline \multirow{4}{*}{ Bombas } & Subsea Multiphase Pump & 115 & 56 & 37 & 29 \\
\cline { 2 - 6 } & Subsea Twin-Screw Pump & 10 & 6 & 4 & 4 \\
\cline { 2 - 6 } & Subsea Helico-Axial Pump & 4 & 3 & 3 & 3 \\
\cline { 2 - 6 } & Subsea Centrifugal Pump & 13 & 7 & 3 & 1 \\
\cline { 2 - 6 } & Electrical Submersible Pump & 39 & 27 & 22 & 18 \\
\hline
\end{tabular}

Fonte: Elaboração própria. Nota: O número de documento apresentado na tabela acima contém dupla contagem, pois não foi feita distinção entre documentos de sistemas tecnológicos e bombas. Nossa intenção é apresentar o número de publicações levantadas por queries.

\section{CARACTERIZAÇÃO BIBLIOMÉTRICA DAS PUBLICAÇÕES ASSOCIADAS BOMBEIO SUBMARINO NA INDÚSTRIA DO PETRÓLEO OFFSHORE}

Esta seção apresenta uma análise bibliométrica das informações coletadas no banco de dados consultado. Com o objetivo de caracterizar a evolução dos conhecimentos difundidos sobre tecnologias de bombeio submarino e seu conteúdo, nossa análise se orienta na seguinte direção, i) evolução temporal das publicações; ii) sua origem geográfica e, finalmente, iii) origem institucional das publicações.

A análise do recorte temporal das publicações tem por objetivo primário observar a trajetória da produção e difusão dos conhecimentos relacionados a um determinado sistema tecnológico, em uma fase précompetitiva. É possível analisar as continuidades e descontinuidades que conformam a trajetória tecnológica do bombeio submarino; no caso específico de nossa investigação, os conceitos tecnológicos tratados nos artigos e trabalhos em anais levantados foram se diversificando com o passar dos anos, expandindo os conteúdos dos conhecimentos produzidos na área. Conforme o gráfico abaixo aponta, o final da década de 2000 demarca o aumento do número de publicações sobre bombeio submarino na indústria do petróleo offshore. A tabela 3, por sua vez, indica que a média de publicações durante as décadas segue a mesma tendência do gráfico 1.

\section{Gráfico 1 - Evolução temporal das publicações científicas associadas ao bombeio submarino no período 1985 - 2015}

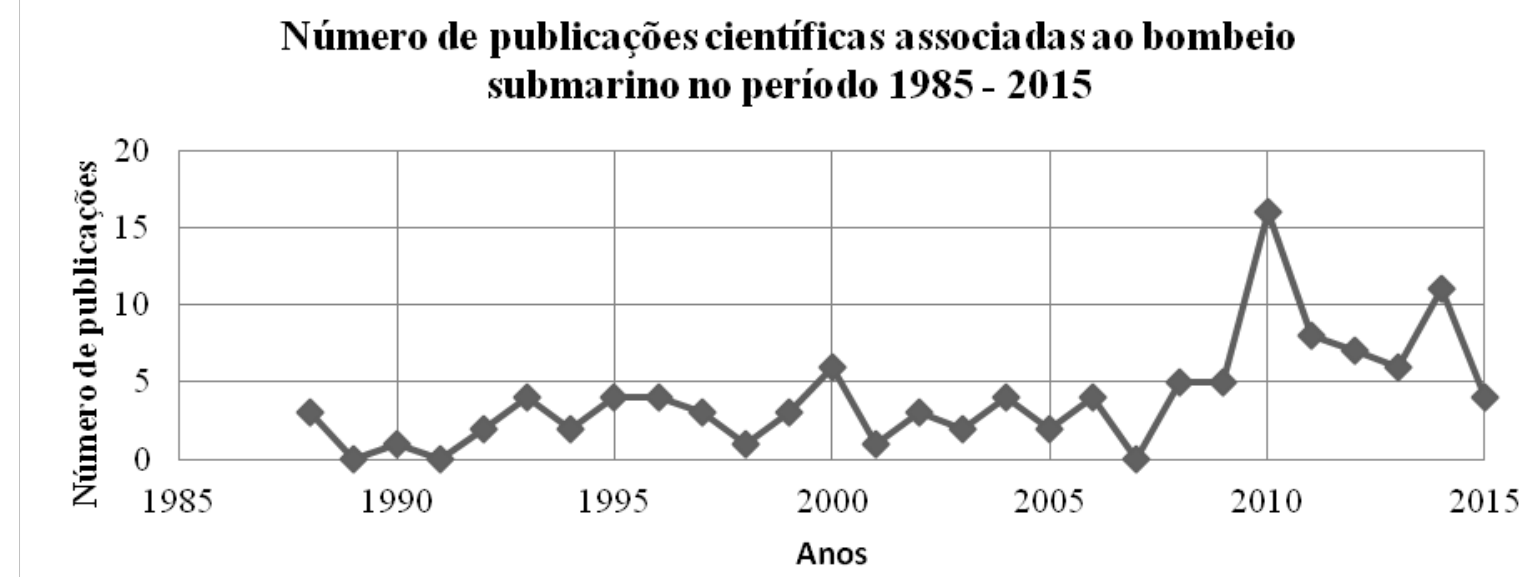

Fonte: Scopus, 2015. 
Tabela 3 - Média de publicações associadas ao bombeio submarino no período 1988 - 2015

\begin{tabular}{|c|c|c|c|}
\hline Período & $\mathbf{N}^{\mathbf{0}}$ de Anos & $\mathbf{N}^{\mathbf{0}}$ de Publicações & Média de Publicações/Anos \\
\hline $\mathbf{1 9 8 8}-\mathbf{1 9 9 0}$ & 3 & 4 & 1,3 \\
\hline $\mathbf{1 9 9 1}-\mathbf{2 0 0 0}$ & 10 & 28 & 2,8 \\
\hline $\mathbf{2 0 0 1}-\mathbf{2 0 1 0}$ & 10 & 43 & 4,3 \\
\hline $\mathbf{2 0 1 1}-\mathbf{2 0 1 5}$ & 5 & 36 & 7,2 \\
\hline
\end{tabular}

Fonte: Elaboração própria.

A análise da evolução temporal das publicações também pode indicar a tendência da frequência da divulgação de novas informações a respeito de uma tecnologia. Uma diminuição pode ser explicada, parcialmente, pelo amadurecimento de certos conceitos tecnológicos ao ponto de passarem do nível do desenvolvimento experimental para o estágio industrial. Neste caso, o compartilhamento dos conhecimentos envolvidos deixa de ser público e passa a ser protegido por seus desenvolvedores, através de diversos mecanismos de proteção de propriedade intelectual como, sobretudo, as patentes (BASSECOULARD; ZITT, 2004). No caso em tela, observa-se, contudo a evolução contrária. O número de publicações aumentou significativamente nas últimas décadas, o que indica que está havendo um importante impulso para a pesquisa em nível experimental. Além disso, por se tratar de uma área em que não se estabeleceu ainda um sistema tecnológico dominante, há competição entre diferentes conceitos inseridos no mesmo paradigma tecnológico.

No que diz respeito à origem geográfica das organizações afiliadas às publicações sobre bombeio submarino (Ver gráfico 2), os dados obtidos foram analisados em duas dimensões, a saber, i) sua distribuição geográfica por grandes regiões do mundo e, posteriormente, ii) raqueamento dos países com maior importância para o endosso às pesquisas na área do bombeio submarino.

As publicações científicas analisadas são afiliadas a organizações de vinte países diferentes. Estes, por sua vez, foram organizados de acordo com sua proximidade geográfica e relações regionais, justificando, desse modo, a divisão regional proposta acima. Não foram registradas afiliações de organizações localizadas no continente africano entre as queries consultadas.

\section{Gráfico 2 - Porcentagem de afiliações das publicações científicas distribuídas segundo regiões do mundo}

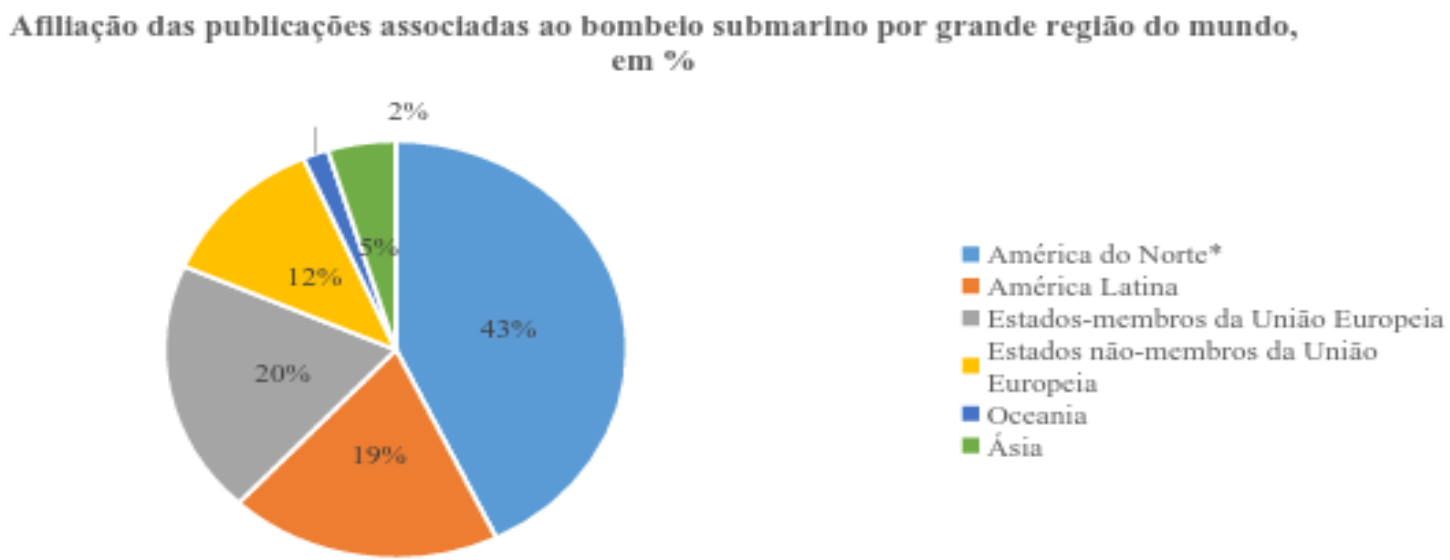

Scopus, 2015. Nota: * - América do Norte não inclui o México.

Fonte:

Como é observável no gráfico acima, a América do Norte (neste caso, Estados Unidos e Canadá) é a região com maior número de organizações que publicam sobre tecnologias de bombeio submarino (43\%), seguida pela América Latina (19\%) e o bloco de países europeus membros da União Europeia (considerando o Reino Unido) (19\%). Se tomarmos como referência, apenas os continentes, a América 
representaria $62 \%$ do total de afiliações das publicações, seguida pelo continente europeu (31\%), Ásia (5\%) e, finalmente, Oceania (2\%). O interessante é associar as regiões com maiores parcelas de organizações afiliadas a publicações àquelas regiões com maior atividade de exploração de hidrocarbonetos offshore, destacando o "peso" do Brasil para a região da América Latina, assim como a origem geográfica das grandes companhias petroleiras e empresas que fornecem serviços e equipamentos à indústria do petróleo. Ou seja, as regiões geográficas com maior número de organizações produzindo conhecimentos sobre bombeio submarino são aquelas que deram origem às grandes players do setor. Essa hipótese fica mais evidente quando analisamos a porcentagem de organizações afiliadas entre os países que mais publicam sobre o bombeio submarino.

\title{
Gráfico 3 - Porcentagem das afiliações das publicações científicas entre os países que mais publicam sobre bombeio submarino
}

\author{
Afiliaçāo das publicaçōes nos quatro paises que mais publicam sobre o bombeio \\ submarino, em \%
}

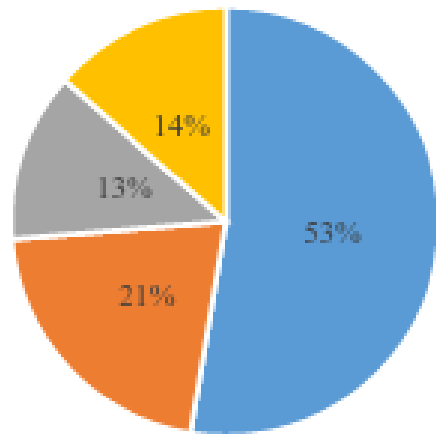

Fonte: Scopus, 2015.

Segundo o gráfico acima, mais da metade do volume das publicações afiliadas aos principais países associados ao desenvolvimento de conhecimentos de bombeio submarino é originário nos Estados Unidos. Desse modo, é possível entender que esse país é o núcleo duro do desenvolvimento de novos conhecimentos sobre bombeio submarino, visto seu maior número de organizações nos setores de engenharia, energia e petróleo, além da sua liderança no processo de inovação. Por sua vez, o Brasil se destaca por seu grande número de projetos no segmento offshore de sua indústria nacional de petróleo e o esforço tecnológico da Petrobrás e de seu centro de pesquisa (CENPES) em identificar e empregar conceitos tecnológicos inovadores que atendam as especificidades de suas operações em águas profundas. Além disso, esses países deram origem às companhias de petróleo que operam, historicamente, nas três regiões de atividade mais intensiva em exploração e produção de petróleo e gás natural offshore com grandes níveis de desafios tecnológicos que marcaram a conformação de três trajetórias tecnológicas distintas, a saber, i) Golfo do México; ii) Mar do Norte e, finalmente, iii) Bacias de Campos e Santos.

O próximo passo de nosso exercício analítico foi classificar as organizações participantes do processo de desenvolvimento do bombeio submarino em três grupos, a saber, i) companhias de petróleo; ii) empresas fornecedoras de equipamentos e serviços e, finalmente, iii) instituições acadêmicas (Universidades, institutos e centros de pesquisa). Os critérios de classificação foram, essencialmente, baseados nas atividades prioritárias das organizações, isto é, a exploração e produção de hidrocarbonetos no primeiro grupo; o oferecimento de serviços complementares e desenvolvimento de novas tecnologias para o setor petrolífero, no segundo grupo e, finalmente, a realização de pesquisa científica. 


\section{Gráfico 4 - Afiliação das publicações científicas por origem institucional, em $\%$ \\ Origem institucional das afiliaçōes das publicaçōes sobre o bombeio submarino, em \%}

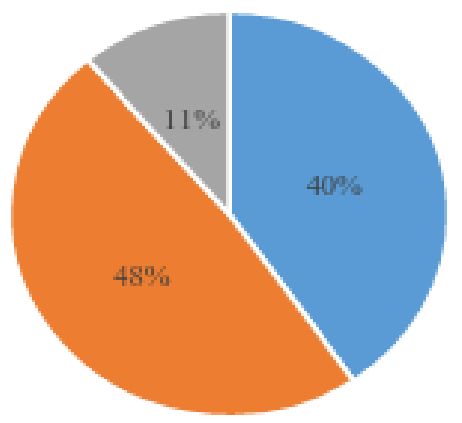

- Companhia de Petróleo

Empresa Fornecedora de Equipamentos c

Servicos

Instituiçāo Acadẽmica

Fonte: Scopus, 2015.

De acordo com as informações apresentadas no gráfico 4, as empresas fornecedoras são o nó da rede de produção de conhecimentos sobre o bombeio submarino (48\% das publicações são afiliadas a uma empresa desse grupo). É notável o fato que $88 \%$ da produção artigos e trabalhos em anais sobre bombeio submarino esteja afiliada a alguma empresa. Fica evidente a importância da P\&D, da pesquisa básica aplicada e da iniciativa empresarial para o desenvolvimento tecnológico nesse setor.

É possível discutir se essa característica confere a esse tipo de produção de conhecimento uma natureza mais relacionada à natureza das tecnologias do que, necessariamente, científica. Isto é, são publicações que apresentam muito mais informações de desempenho dos conceitos tecnológicos que, necessariamente, uma discussão das bases científicas que os sustentam. Em realidade, isso se explica pelo fato de artigos relacionados à área de engenharia e afins estarem muito mais preocupados com a socialização de suas experiências com a aplicação de tecnologias, sobretudo quando são afiliados a corporações que buscam aperfeiçoar resultados, ou identificar conceitos tecnológicos interessantes às suas atividades econômicas. A elevada participação de empresas neste tipo de produção também indica a preocupação das organizações em difundir conhecimentos em uma fase pré-competitiva, isto é, quando as trocas de experiências são muito importantes para fazer avançar o conhecimento sobre determinada tecnologia (FREEMAN, 1991; GLÄNZEL; SCHUBERT, 2004; HERTZFELD; LINK; VONORTAS, 2006).

Um dos objetivos de nossa investigação foi identificar as organizações que mais publicam sobre bombeio submarino. O gráfico 5 confirma a importância das empresas na produção de novos conhecimentos tecnológicos no que diz respeito a essas tecnologias. O ranqueamento das dez organizações com maior número de artigos e trabalhos em anais aponta que a Petrobrás é a companhia de petróleo com maior número de publicações (19), destacando a importância do Brasil e dessa empresa para o desenvolvimento dessas tecnologias. Isso não significa, necessariamente, que o país seja responsável pela produção de novas tecnologias associadas ao bombeio submarino, isto é por inovações de ruptura, mas sim que é estratégico à aplicação desses conceitos tecnológicos em testes experimentais de novos métodos de produção e recuperação de hidrocarbonetos. Além disso, o conteúdo dos artigos afiliados a Petrobrás é explicitamente descritivo das experiências e da performance das tecnologias de bombas operando nas condições específicas da costa brasileira.

\section{Gráfico 5 - Organizações que mais publicam sobre bombeio submarino}




\title{
Número de publicações sobre o bombeio submarino afliadas às
} organizações



\author{
- Petrobras \\ - Shell \\ aBB \\ - Chevron \\ - Statoil \\ -FMC Technologies \\ - Total \\ -Aker \\ - ConocoPhillips \\ - Baker Hughes
}

Fonte: Scopus, 2015.

\section{REDES COOPERATIVAS DE PESQUISA TECNOLÓGICA NA INDÚSTRIA DO PETRÓLEO OFFSHORE: O CASO DO BOMBEIO SUBMARINO}

O estabelecimento de parcerias em áreas de fronteira tecnológica é um instrumento utilizado pelas empresas para partilhar riscos e custos, envolvendo certo nível de cooperação estratégica e operacional para alcançar um objetivo comum às organizações (TEECE, 1992). Esse fenômeno, crescente desde a década de 1980, implicou em novas formas de cooperação entre organizações para o desenvolvimento de novos conhecimentos e inovações (HERTZFELD; LINK; VONORTAS, 2006). Mesmo em áreas de pouco dinamismo tecnológico como a indústria do petróleo, esse mecanismo é usado por companhias de petróleo, empresas fornecedoras de bens e serviços, instituições de pesquisa e universidades para avançar em áreas de fronteira como a exploração e produção de petróleo em lâminas d'água cada vez mais profundas.

Ao analisar os vínculos de cooperação entre as organizações que produzem novos conhecimentos sobre o bombeio submarino, podemos descrever a rede de cooperação de pesquisa tecnológica que permitiu expandir e maturar essas tecnologias. Para fins metodológicos, entendemos neste trabalho que a cooperação de pesquisa científica e tecnológica ocorre na fase pré-competitiva de um dado sistema tecnológico, permitindo que a análise bibliométrica sobre artigos em co-autoria afiliados a mais de uma organização possa evidenciar o padrão de relacionamento inter-institucional e os nós dessa rede de produção de conhecimentos (FREEMAN, 1991; BASSECOURLARD; ZITT, 2004; GLÄNZEL; SCHUBERT, 2004). Neste artigo não nos propomos a analisar as parcerias estratégicas estabelecidas entre as organizações que depositam patentes dessas tecnologias de bombeio submarino, pois as consideramos um fenômeno da fase competitiva da inovação. Não entramos neste escopo, todavia, há intenções em analisar, em uma pesquisa futura, as patentes associadas ao bombeio submarino com o objetivo de desenhar a rede de parcerias que se constitui nessa área.

No que diz respeito à rede de cooperação de pesquisa tecnológica sobre o bombeio submarino, elaboramos seis tipos de vínculos cooperativos possíveis entre as organizações, a saber, i) entre companhias de petróleo e empresas fornecedoras; ii) entre companhias de petróleo e instituições acadêmicas; iii) entre companhias de petróleo; iv) entre empresas fornecedoras e instituições acadêmicas; v) entre empresas fornecedoras e, finalmente, vi) entre instituições acadêmicas. A classificação dos vínculos cooperativos proposta (Ver Gráfico 6) se baseou na identificação da origem institucional dos autores de publicações em co-autoria afiliadas a mais de uma organização. A tabela 4 apresenta a relação 
do número de publicações em cooperação da nossa amostra. No caso, 35 artigos ou trabalhos em anais (31,5\%) foram publicados em algum tipo de cooperação de pesquisa.

\section{Gráfico 6 - Cooperação de pesquisa entre as organizações que publicam sobre bombeio submarino, em \%}

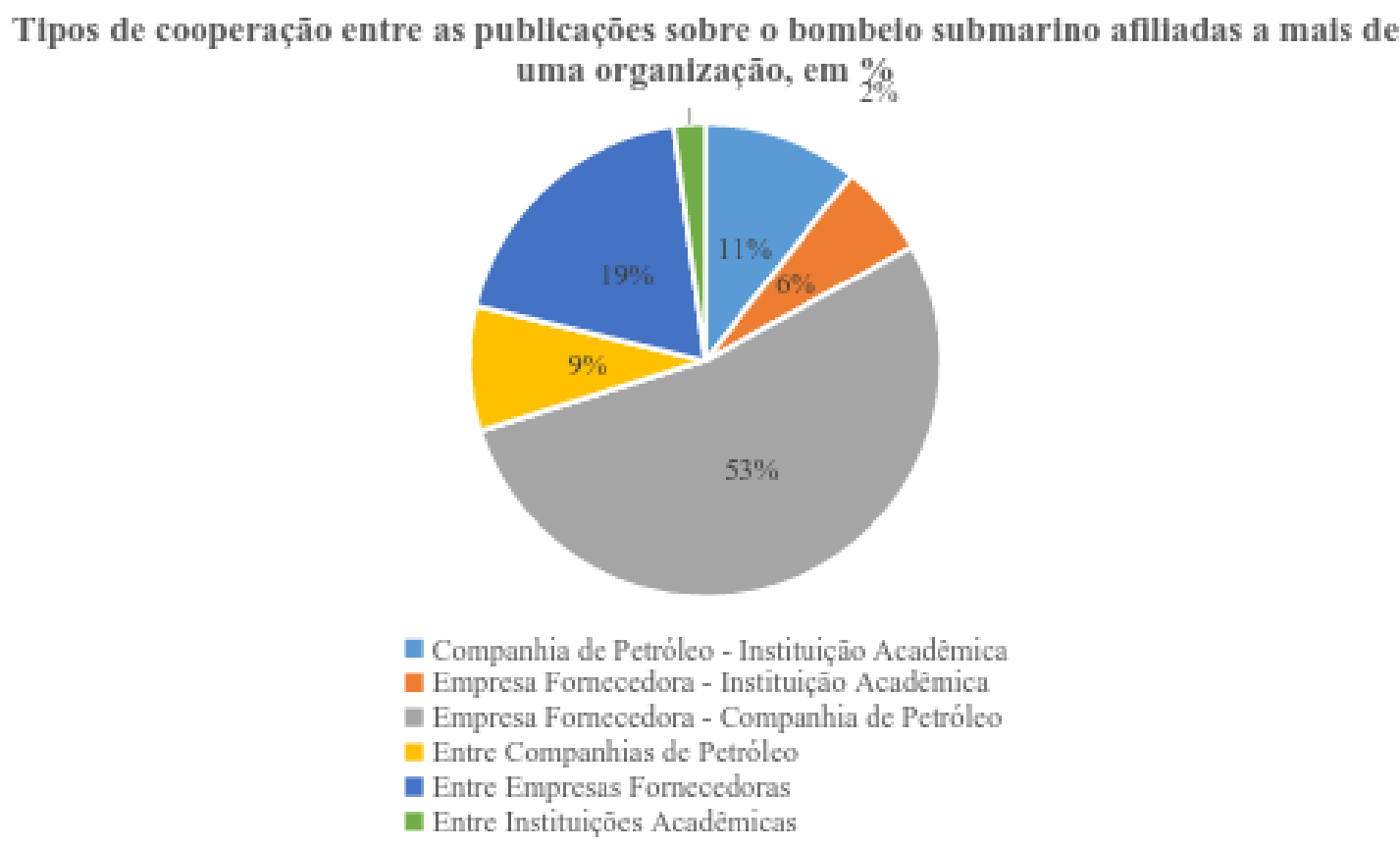

Fonte: Elaboração própria.

Tabela 4 - Publicações em cooperação de pesquisa em relação ao total de publicações associadas ao bombeio submarino na base Scopus no período 1988 - 2015 , em \%

Fonte: Elaboração própria.

\begin{tabular}{|c|c|c|}
\cline { 2 - 3 } \multicolumn{1}{c|}{} & $\mathbf{N}^{\mathbf{0}}$ de Publicações & $\mathbf{N}^{\mathbf{0}}$ de Publicações (\%) \\
\hline Total de Publicações & 111 & 100 \\
\hline Publicações em cooperação inter-organizacional & 35 & 31,5 \\
\hline Publicações sem cooperação inter-organizacional & 76 & 68,5 \\
\hline
\end{tabular}

Este dado é interessante por evidenciar o padrão de relacionamento das organizações envolvidas com o processo de inovação e desenvolvimento de novos conhecimentos sobre tecnologias de bombeamento submarino. No caso estudado, 53\% das publicações são em cooperação de pesquisa entre companhias de petróleo e empresas fornecedoras de equipamentos e serviços, caracterizando um tipo de relacionamento usuário - fornecedor. Além disso, constata-se que as empresas fornecedoras são o nó principal da rede de relacionamento, visto que em $78 \%$ dos vínculos cooperativos nessa rede envolvem alguma empresa dessa categoria. Esta ideia refuta a reflexão inicial de que as companhias de petróleo cooperam mais entre si para desenvolver inovações e contribuições tecnológicas para o bombeio submarino. Contudo, as companhias de petróleo ainda se apresentam como importantes articuladoras nessa fase do processo de inovação.

O que chama mais nossa atenção nesse recorte analítico são os comportamentos mais ou menos colaborativos das organizações para desenvolver conhecimentos a respeito das tecnologias de bombeamento submarino. Nesse sentido, fica justificado que instituições acadêmicas tenham um papel secundário no caso do bombeio submarino, devido ao fato dessa área estar ligada à pesquisa de alta intensidade tecnológica e não muito orientada à pesquisa científica. Por sua vez, as companhias de petróleo e gás, assim como as empresas fornecedoras mais inclinadas a cooperar para produzir novos 
conhecimentos, devido os desafios tecnológicos e as barreiras à produção de conhecimento conduzida individualmente. É destacado aqui que $98 \%$ das publicações em cooperação de pesquisa sobre bombeio submarino envolvem alguma empresa.

\section{Gráfico 7 - Organizações que mais publicam em cooperação sobre bombeio submarino}



Fonte: Scopus, 2015.

As informações expostas acima identificam quais organizações mais publicam artigos ou trabalhos em anais em cooperação de pesquisa tecnológica. Neste caso, nota-se que as companhias de petróleos são as organizações que mais publicam em cooperação. A Petrobrás apresenta uma característica menos inclinada à produção de conhecimentos tecnológicos de modo mais colaborativo, visto que das 19 publicações afiliadas a essa empresa, apenas 3 foram elaboradas cooperativamente, isto é, 15,8\%. A Shell, por sua vez, contabiliza 83,3\% de publicações em co-autoria afiliadas a outras organizações. Desse modo, argumentamos que à Petrobrás cabe um papel de “campo de testes” para as tecnologias emergentes na área do bombeio submarino.

\section{CONSIDERAÇÕES FINAIS}

A adoção em larga escala da tecnologia do bombeio submarino ainda é limitada devido à complexidade dos grandes desafios que implicam a imersão a grandes profundidades de equipamentos de grande porte. Considerando o volume da produção conhecimentos associado a essas tecnologias, entende-se que ainda se encontram em uma fase de maturação pautada pelo aporte de pesquisas colaborativas entre organizações.

O Brasil se destaca na publicação de conhecimentos associados ao bombeio submarino, mostrando-se estratégico para a avaliação de desempenho de inovações tecnológicas no segmento offshore da indústria do petróleo. Somente o Brasil detém 21\% de todas as publicações entre os quatro países com maior número de artigos e anais de congressos no tema. Isto quer dizer que a representatividade da produção científica brasileira é alta no desenvolvimento de conhecimentos utilizados para o desenvolvimento de novas tecnologias de bombeio submarino. A liderança da Petrobrás, como a companhia de petróleo com maior número de trabalhos científicos no conjunto de nossa amostra reforça o argumento da importância do Brasil para o bombeio submarino. Todavia, a empresa é considerada como um importante locus de aplicação de conceitos tecnológicos experimentais e de testes destes.

Numa perspectiva bibliométrica, a análise realizada indicou que na área do bombeio submarino artigos e trabalhos em anais tratam da produção de conhecimentos mais associados à experiência e à natureza tecnológica dos conceitos que conhecimentos científicos. Além disso, as organizações tendem a 
apresentar comportamentos mais colaborativos no nível pré-competitivo que competitivo do desenvolvimento tecnológico. Essa característica é justificada pelo fato das organizações compartilharem experiências na aplicação de tecnologias no nível de publicações, sendo essa etapa fundamental para o impulso à pesquisa e inovação das organizações, enquanto que no nível de patentes há preocupação em proteger os conhecimentos tecnológicos adquiridos.

As companhias de petróleo, empresas fornecedoras de equipamentos e serviços e organizações de pesquisa são as organizações que impulsionam o bombeamento submarino, destacando que $98 \%$ das publicações em cooperação de pesquisa sobre bombeio submarino envolvem alguma empresa. Em sua fase pré-competitiva, as principais relações entre as organizações são do tipo usuário - fornecedor; neste caso, os vínculos colaborativos entre companhias de petróleo e empresas fornecedoras de equipamentos e serviços correspondem a 53\% das pesquisas em cooperação tecnológica encontradas. Com o objetivo de diminuir incertezas e riscos derivados de uma tecnologia ainda em fase experimental, além de compartilhar os custos do processo de desenvolvimento tecnológico, as pesquisas em cooperação tecnológica estabelecidas entre instituições se tornaram um recurso muito valioso para a inovação e difusão de conhecimentos em rede. O bombeio submarino vem se tornando uma realidade à medida que novos projetos colaborativos corroboram o seu amadurecimento, cogitando, em médio prazo, um breakthrough da trajetória tecnológica da indústria do petróleo.

\section{BIBLIOGRAFIA}

ARTHUR, Brian. Competing technologies, increasing returns, and lock-in by historical events. In: The Economic Journal, 99, 394, 116 - 131. 1989.

BASSECOULARD, Elise; ZITT, Michel. Patents and publications. In: MOED, H. F. et al. (Eds.). Handbook of Quantitative Science and Technology Research. New York: Kluwer Academic Publishers, 665 - 694. 2004.

BRITISH PETROLEUM. BP Energy outlook 2015. 2015. Disponível em: $<$ http://www.bp.com/content/dam/bp/pdf/energy-economics/energy-outlook-2015/bp-energy-outlook2035-booklet.pdf $>$ Último acesso em: 12 de janeiro de 2016.

DOSI, Giovanni. Technological paradigms and technological trajectories. In: Research Policy, 11, 142 167. 1982.

ELSEVIER. Scopus content coverage guide. 2016. Disponível em: $<$ https://www.elsevier.com/_data/assets/pdf_file/0007/69451/scopus_content_coverage_guide.pdf>

Último acesso em: 05 de junho de 2016.

FALCONE, Gioia et al.. Multiphase flow metering: Current trends and future developments. In: Distinguished Author Series. 2002, 77 - 84. 2002.

FREEMAN, Christopher. Networks of innovators: A synthesis of research issues. In: Research Policy, 20, 5, oct 1991, 499 - 514. 1991.

FURTADO, Andre Tosi; FREITAS, Adriana Gomes de. The catch-up strategy of Petrobrás through cooperative R\&D. In: Journal of Technology Transfer, 25, 23 - 36. 2000.

Cooperação tecnológica e arranjos contratuais: Uma análise das parcerias de P\&D da Petrobrás, 01/2001, CIENTÍfICO NACIONAL, II Seminário Brasileiro de Nova Economia Institucional, Vol. 1, 57 - 78, Campinas, SP, BRASIL. 2001. 
GLÄNZEL, Wolfgang.; SCHUBERT, András.. Analysing scientific networks through co-authorship. In: MOED, H. F. et al. (Eds.). Handbook of Quantitative Science and Technology Research. New York: Kluwer Academic Publishers, 257 - 276. 2004.

HERTZFELD, Henry.; LINK, Albert.; VONORTAS, Nicholas. Intellectual property protection mechanisms in research partnerships. In: Research Policy, 35, (6), 825 - 838. 2006.

HUA, Gong et al.. Comparison of multiphase pumping technologias for subsea and downhole applications. In: Oil and Gas Facilities, feb, 2012, 36 - 46. 2012.

OFFSHORE MAGAZINE. 2015 Worldwide survey of subsea processing: Separation, compression and pumping systems. 2015. Disponível em: < http://www.offshore-mag.com/content/dam/offshore/printarticles/volume-75/03/1503off_SubseaposterDIG.pdf> Último acesso em: 20 de abril de 2016.

ROMERO, Oldrich J.; HUPP, Anderson. Subsea electrical submersible pump significance in petroleum offshore production. In: Journal of Energy Resources Technology, 136, 1, 1 - 8. 2013.

TEECE, David. Competition, cooperation and innovation: Organizational arrangements for regimes of rapid technological progress. In: Journal of Economic Behavior and Organization, 18. 1992.

VONORTAS, Nicholas.; SAFIOLEAS, Stratos. Strategic alliances in information technology and developing country firms: Recent Evidence. In: World Development, 25, 5, 657 - 680. 1997. 